\title{
FREQUENCY OF THROMBOCYTOPENIA AND ITS SEVERITY IN PATIENTS OF MALARIA.
}

\footnotetext{
1. MBBS, FCPS Hematology

Assistant Professor

Department of Pathology Nishtar Medical University \& Hospital, Multan.

2. MBBS, M.Phil. Hematology

Assistant Professor

Department of Pathology Nishtar Medical University \& Hospital, Multan

3. MBBS

Demonstrator Community medicine Rawalpindi Medical University Rawalpindi

4. MBBS, M.Phil. Hematology Assistant Professor

Department of Pathology Ibn e Sina Hospital,

Multan Medical \& Dental College, Multan.

5. MBBS, M.Phil. Hematology

Assistant Professor

Department of Pathology Rahber Medical College, Lahore.

6. MBBS, FCPS Hematology Assistant Professor

Department of Pathology Govt. Khawaja Muhammad Safdar Medical College, Sialkot.

Correspondence Address:

Dr. Yasmeen Batool

Department of Pathology

Nishtar Medical University \& Hospital

Multan.

dryasmeenbatool@gmail.com
}

Article received on:

$08 / 11 / 2018$

Accepted for publication:

$18 / 02 / 2019$

Received after proof reading:

28/08/2019

\begin{abstract}
Yasmeen Batool', Sabeen Fatima², Gulzaib Pervaiz ${ }^{3}$, Naseem Akhter ${ }^{4}$, Maliha Asif ${ }^{5}$, Faisal Bashir ${ }^{6}$
ABSTRACT: Pakistan is an endemic area for malaria. Malaria is considered to be associated with thrombocytopenia. The frequency of thrombocytopenia varies in different studies. This study was done to determine the frequency of thrombocytopenia among patients with malaria. Objectives: To determine the frequency and severity of thrombocytopenia in patients of malaria. Study Design: Cross-sectional survey. Setting: Pathology Department, Nishtar Medical University, Multan. Period: Eight months; (04-03-2018 to 03-11-2018). Materials \& Methods: One hundred and fifty patients with malaria were included in the study. Platelet count of all the patients was done on Sysmex KX-21. The patients were categorized according to the presence of thrombocytopenia (yes or no) and the severity of thrombocytopenia (mild, moderate or severe). The data was described as frequency distribution table. Results: The mean age of the patients was $31.81+15.31$ years. There were $46(30.7 \%)$ female patients and $104(69.3 \%)$ male patients. Thrombocytopenia was present among 103 (68.7\%) patients. Mild, moderate and severe thrombocytopenia was found in 60 (58.3\%), 24 (23.3\%) and 19 (18.4\%) patients, respectively. Conclusion: This study concludes that frequency of thrombocytopenia is very high among patients with malaria. Severity of thrombocytopenia is mild to moderate, with few patients presenting with severe thrombocytopenia. This finding signifies that malaria is an important cause of thrombocytopenia. So the platelet count should be monitored closely in patients of malaria to avoid possible complications of thrombocytopenia. Moreover, it is suggested that malaria is an important differential diagnosis of thrombocytopenia with fever but needs confirmation by thick and thin smears for malarial parasite. In case of viral infections like dengue fever, smears will be negative for malarial parasite and patients will present with leucopenia and thrombocytopenia and majority of peripheral blood leucocytes will be reactive lymphocytes.
\end{abstract}

Key words: Malaria, Thrombocytopenia, Vector, Plasmodium, Platelets.

Article Citation: Batool Y, Fatima S, Pervaiz G, Akhter N, Asif M, Bashir F. Frequency of thrombocytopenia and its severity in patients of Malaria. Professional Med J 2019; 26(9):1398-1403. DOI: 10.29309/TPMJ/2019.26.09.691

\section{INTRODUCTION}

Malaria is a vector born disease caused by the bite of the female Anopheles mosquito inoculating the sporozoites in the human blood stream leading to clinical manifestations. ${ }^{1}$ Malaria affects approximately $5 \%$ of the world population, being endemic in 103 countries and causes 1-3 million deaths each year. About 270 million people are infected each year. ${ }^{2}$ WHO forecasts a $16 \%$ growth in annual cases of malaria. ${ }^{3,4}$

In Pakistan, half a million malaria cases occur annually and mortality rate is as high as 50 thousand deaths per year, mostly affecting infants, children and pregnant ladies. ${ }^{5}$ Malaria can be caused by any of four parasites of plasmodium species namely Plasmodium Vivax, Plasmodium Falciparum, Plasmodium ovale, and Plasmodium malariae, out of which Plasmodium Falciparum is the main species responsible for the most of these deaths. ${ }^{6}$ Early diagnosis remains the key for the effective management of malaria for a favorable outcome. ${ }^{7}$

Clinically malaria mimics many diseases and there are no absolute diagnostic clinical features. Unfortunately, most of the diagnostic facilities are not available in all areas endemic for malaria. In such settings changes in hematological parameters can be of great help to support the 
diagnosis. The presence of thrombocytopenia in a patient living in endemic area has become a highly sensitive diagnostic marker with $60 \%$ sensitivity and $88 \%$ specificity for the diagnosis of malaria. The speculated mechanisms leading to thrombocytopenia in malaria are: hypersplenism, bone marrow depressions, coagulation disturbances, antibody mediated platelet destruction, oxidative stress, and the role of platelets as cofactors in triggering severe malaria. $^{8}$

Thrombocytopenia is one of the most common complications of both Plasmodium vivax and Plasmodium falciparum malaria ${ }^{9}$, defined as platelet count less than $150 \times 10^{\%} / \mathrm{L}$. It is divided into three main categories; mild (100 to $150 \times 10^{9} / \mathrm{L}$ ), moderate (50 to $99 \times 10^{9} / \mathrm{L}$ ), and severe $\left(<50 \times 10^{9} / \mathrm{L}\right) .^{10}$

Frequency of thrombocytopenia in malaria is found to be $70 \%$. Mild thrombocytopenia is observed in $70 \%$ cases, moderate in $22 \%$ and severe in $8 \%$ cases. ${ }^{11}$ Thrombocytopenic malaria, in contrast to the non-thrombocytopenic ${ }^{12}$ variety, correlates with a higher degree of parasitemia and increased cytokine production. ${ }^{13}$ Bleeding in thrombocytopenia is however not common, and occurs in less than $5-10 \%$ of cases of severe malaria. ${ }^{14,15}$ Thrombocytopenia is considered to be an important predictor of severity of childhood Falciparum malaria. ${ }^{16}$

In developing countries where malaria is endemic, no such study has been conducted before with special emphasis on thrombocytopenia and its degree of severity. Determination of frequency of thrombocytopenia in malaria endemic area will help in establishing malaria as an important cause of thrombocytopenia and will help clinicians in early diagnosis of these malaria patients. This study will also help in determining degree of severity of thrombocytopenia associated with malaria. This will assist in formulating guidelines and management plan for treatment of this grave complication of malaria according to its degree of severity.

\section{OBJECTIVES}

The objectives of this study were:

1. To determine the frequency of thrombocytopenia in patients of malaria.

2. To determine the severity of thrombocytopenia in patients of malaria.

\section{MATERIAL AND METHODS}

After approval of study by ethical board of the institution. A total of 150 patients of both genders ranging from 12-80 years with a history of fever less than seven days duration with no localizing signs and presence of malarial parasite in the peripheral blood film presenting to Pathology laboratory of Nishtar Medical University, Multan over a period of 8 months duration, from $04 / 03 / 2018$ to $03 / 11 / 2018$, were included in the study. Patients with malarial parasite in peripheral blood smear but with other causes of thrombocytopenia e.g.; marrow failure, marrow infiltration, ITP, chronic liver disease, bleeding disorders, drug intake such as septran, thiazides and chemotherapeutic agents assessed through history, bone marrow examination were excluded from the study, because in these conditions cause of thrombocytopenia was not clear.

After explaining patients about pros and cons of the procedure and taking informed consent and ensuring their confidentiality, three milliliter venous blood was collected in EDTA vacutainers, from each patient. Thick and thin blood smears were prepared from each specimen, stained with Giemsa stain and examined for malarial parasite under light microscope using $100 \times$ oil immersion lens. Complete blood counts of each specimen of all malaria cases were performed by Sysmex KX-21 automated hematology analyzer and platelet count of all cases were noted. Platelet counts of malaria patients having thrombocytopenia were also re-assessed on Giemsa stained blood smear by light microscope using $100 \times$ oil immersion lens in Pathology department of Nishtar Medical University, Multan. Effect modifiers were Plasmodium species and were dealt by stratification. Data was analyzed on SPSS version 20. Quantitative variables like patient's age were analyzed by mean \pm standard deviation. Qualitative variables including the 
gender and the presence of thrombocytopenia and its severity were presented in the form of frequencies and percentages. Chi square test was used to analyze the difference between two groups and $P$ value equal or less than 0.05 was considered as significant.

\section{RESULTS}

Out of 150 cases, 46 were (30.7\%) female and $104(69.3 \%)$ male patients with female to male ratio of $1: 2.3$.There were $43(28.7 \%)$ patients of age $12-20$ years, $31(20.7 \%)$ patients in the age group 21 - 30 years, 32 (21.3\%) patients in the age group $31-40$ years, $29(19.3 \%)$ patients of age group 41 - 50 years, $10(6.7 \%)$ patients of age group 51 - 60 years, 5 (3.3\%) patients of age group $61-70$ years and none of the patients included in age group $71-80$ years. The mean age of the patients was $31.8 \pm 15.3$ years. The age range was from $12-68$ years. Plasmodium vivax was present among 138 (92\%) patients while plasmodium falciparum in $12(8 \%)$ patients. (Figure-1)

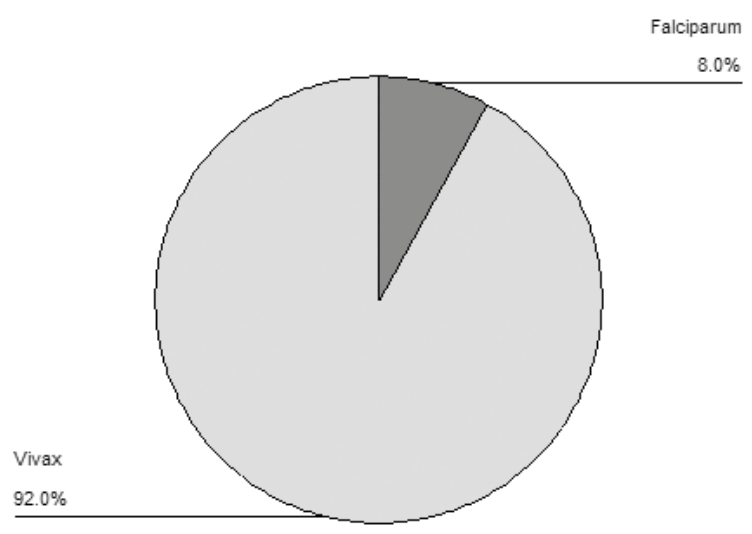

Figure-1. Distribution of patients by type of malarial parasite $(n=150)$

The mean thrombocyte count in study population was $125.98 \pm 63.916 .56 \times 10^{9} / \mathrm{L}$ (range $3-320$ $\left.\mathrm{X} 10^{9} / \mathrm{L}\right)$. Among the 150 patients included in the study, thrombocytopenia was present among $103(68.7 \%)$ patients, while $47(31.3 \%)$ patients had normal platelet count. (Figure-2)

Out of 103 patients who had thrombocytopenia, 93 patients were infected with P. vivax and 10 infected with P. Falciparum (Table-I).

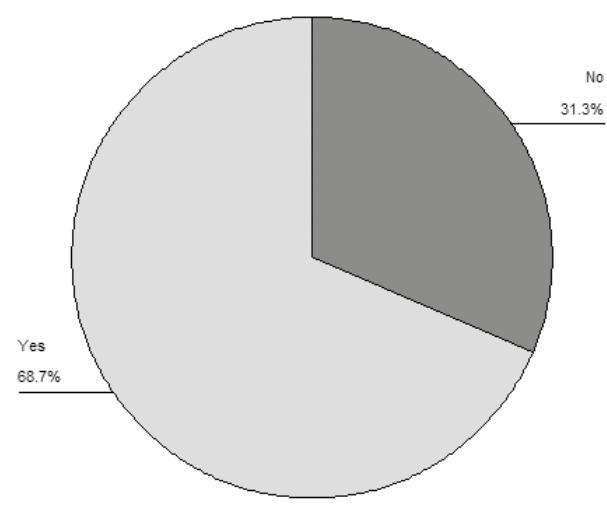

Figure-2. Frequency of thrombocytopenia in total patients $(n=150)$.

\begin{tabular}{|l|c|c|c|c|}
\hline \multirow{2}{*}{$\begin{array}{c}\text { Total } \\
\text { Patients }\end{array}$} & \multicolumn{3}{|c|}{$\begin{array}{c}\text { Severity of } \\
\text { Thrombocytopenia }\end{array}$} & (P-Value) \\
\cline { 2 - 4 } & Mild & Moderate & Severe & \\
\hline & No. & No. & No. & \\
P. Vivax & $\%$ & $\%$ & $\%$ & \\
n=93 & 58 & 20 & 15 & \\
\cline { 2 - 4 } & $(63)$ & $(21)$ & $(16)$ & $(0.032)^{a}$ \\
\hline P. & 02 & 04 & 04 & \\
Falciparum & $(20)$ & $(40)$ & $(40)$ & \\
$n=10$ & & & & \\
\hline
\end{tabular}

Table-I. Severity of thrombocytopenia in patients of

Plasmodium Vivax and Plasmodium Falciparum. a $p$ value $\leq \mathbf{0 . 0 5}$ was considered significant.

$60(58.3 \%)$ patients were categorized as having mild thrombocytopenia, 24 (23.3\%) patients had moderated thrombocytopenia and 19 (18.4\%) patients had severe thrombocytopenia. (Figure-3)

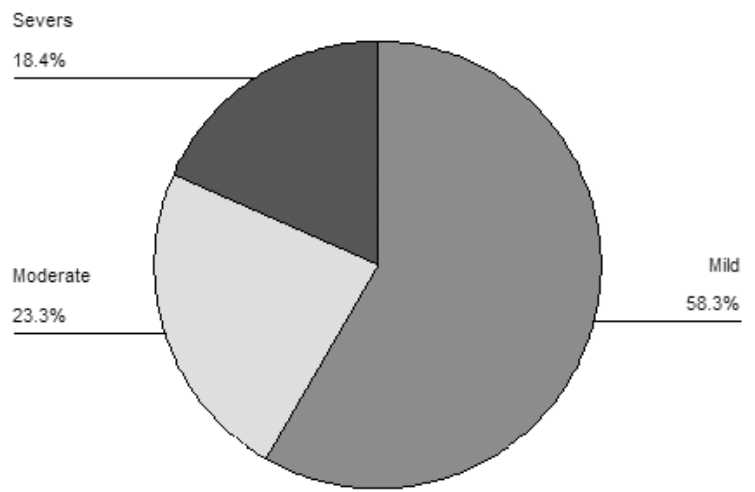

Figure-3. Severity of thrombocytopenia in patients presenting with thrombocytopenia $(n=103)$. 


\section{DISCUSSION}

In this study, we included 150 patients of malaria to determine the frequency and severity of thrombocytopenia. This was a prospective crosssectional survey which was conducted in a wellequipped tertiary care unit.

The mean age of the patients in our study was $31.81 \pm 15.31$ years. While in another study by Jadhav, et al, ${ }^{17}$ the mean age of patients was 37.4 \pm 14.2 years. In a local study by Ansari $S$, et al, ${ }^{5}$ the mean age was $34 \pm 1.7$ years (range 16-53 years). In another local study by Shaikh QH, et al, 7 the mean age of the patients was $28.9 \pm 15.42$ years. In our study, the highest percentages of patients were of $<20$ years. Younger people have less immunity to malarial parasite in endemic areas. The results of our study showed a high frequency of thrombocytopenia in our patients i.e. $68.7 \%$. Majority of our patients are suffering from plasmodium vivax. Plasmodium vivax is more associated with mild decrease in platelet count.

This is in contradiction to the study by Bhandary et $\mathrm{al}^{18}$, which showed that malaria was present in only $11.76 \%$ female patients while majority (67.64\%) of the patients belonged to age group $20-45$ years. This reflects that age of patients presenting with varies greatly among different populations.

The majority of the patients in our study were males i.e. $69.3 \%$. This observation is in agreement with studies by different authors. Studies by Bhandray et $\mathrm{al}^{18}$, Shaikh et $\mathrm{al}^{7}$ and Ansari et $\mathrm{al}^{5}$ showed $77.45 \%, 72.5 \%$ and $70.2 \%$ patients were males respectively. However, Jadhav et $\mathrm{al}^{17}$, documented a small difference in male and female population suffering from malaria, although male population was dominated. In their study, they found that $58.5 \%$ patients were males and $41.5 \%$ were females. The trend in literature reflects that malaria is seen more frequently among male as compared to female population possibly because females in this area have less access to medical facilities to get diagnosed due to poverty, socio economic issues and ignorance.
In our study, 92\% patients had Plasmodium vivax infection and plasmodium falciparum was detected in $8 \%$ patients. Jadhav UM, et al in their study on a large sample size of 1565 patients, found a higher frequency of Plasmodium vivax patients but the difference was not large, like in our study. They found Plasmodium vivax in $62.2 \%$, and plasmodium falciparum in $37.7 \%$ while mixed in $0.1 \%$ patients. In other Indian study conducted by Bhandry et al, $49.01 \%$ patients had mixed malaria \& $45.09 \%$ were positive for Plasmodium vivax. Isolated P. falciparum was detected in only $5.88 \%$. In another local study by Shaikh et al, it was found that $51.60 \%$ patients were suffering with Plasmodium falciparum and $48.40 \%$ patients had Plasmodium vivax infection. This reflects that Plasmodium vivax and plasmodium falciparum is seen in different frequencies. Some studies have shown higher and some have shown lower percentage of $P$ falciparum infection. The frequency of plasmodium falciparum infection is low in our area.

The mean platelet count in our study was 125.98 $\pm 63.91 \times 10^{9} / \mathrm{L}$. This was very close to that of study by Jadhav et al, which showed that mean platelet count was $1,15 \times 10^{9} / \mathrm{L}( \pm S D$ 64,580) with a range of $8000-5,73 \times 10^{9} / \mathrm{L}$. The mean platelet count in study by Ansari S, et al was $12 \times 10 \pm 450$ $\mathrm{x} 10^{9} / \mathrm{L}$. Our patients suffering from plasmodium vivax did not show a very low mean platelet count. This may be due to the reason that Plasmodium falciparum is notorious for causing a more drop in platelet count as compared to vivax and the frequency of Plasmodium falciparum in our study was very low. The mean platelet count in patients affected with P falciparum was $100,900 / \mu \mathrm{l}( \pm$ SD $75,437)$. So, there is a more drop in platelet count in patients with $\mathrm{P}$ falciparum group.

In our study, $68.7 \%$ patients suffered from thrombocytopenia. This is in accordance with other studies. In a study by Zaid $\mathrm{HA}^{19}$, conducted at Qatar, which included 78 patients infected with Plasmodium vivax, the frequency of thrombocytopenia was $83.3 \%$. This is a very high frequency of thrombocytopenia. In a large trial including 1565 patients, Jadhav, et al documented that frequency of thrombocytopenia was $78.4 \%$ 
of the cases. The frequency of thrombocytopenia as determined by Ansari S, et al was $69.18 \%$. In a study by Rodríguez-Morales, et al, ${ }^{20}$ a total of 116 patients with P. vivax malaria were included and the frequency of thrombocytopenia was $65 \%$. Shaikh QH, in a study of 120 patients with malaria found that frequency of thrombocytopenia was $80.1 \%$. This was a high frequency than our study. This may be due to the reason that in their study, they had a higher frequency of Plasmodium falciparum i.e. $53.3 \%$.

In our study, mild thrombocytopenia was the most common i.e. present in $58.3 \%$ patients followed by moderate thrombocytopenia (23.3\%) and severe thrombocytopenia $18.4 \%$ patients. However, this distribution was different in other studies. Ansari S, et al, documented that mild, moderate and severe thrombocytopenia was found in $10.5 \%, 48.6 \%$ and $10 \%$ patients, respectively. Zahid $\mathrm{HA}$, et al, documented that $52.6 \%$ had mild thrombocytopenia, $29.5 \%$ had moderate thrombocytopenia and $1.3 \%$ patients had severe thrombocytopenia. Rodríguez-Morales, et al, described that $43 \%$ of the thrombocytopenic population in their study suffered from severe thrombocytopenia. In our study, we observed that Plasmodium Falciparum is associated with a more drop in platelet count as compared to Plasmodium Vivax ( $P$ value $=0.032)$. It is very close to study by Jadhav UM, et al, who also noted significant association between severity of thrombocytopenia and type of malaria.

This study had some limitations. This was single center study with small sample size. This study was conducted in a tertiary care hospital where most of the patients come to the hospital with serious or complicated problems rather for fever or malaria. Due to poverty and ignorance in our population, most of the time, the problems remain undiagnosed or maltreated. So, the frequency of thrombocytopenia may be higher than observed in our study.

\section{CONCLUSION}

This study concludes that frequency of thrombocytopenia is very high among patients with malaria. Severity of thrombocytopenia is mild to moderate, with few patients presenting with severe thrombocytopenia. This finding signifies that malaria is an important cause of thrombocytopenia. So, the platelet count should be monitored closely in patients of malaria to avoid possible complications of thrombocytopenia.

\section{Copyright $@ 18$ Feb, 2019.}

\section{REFRENCES}

1. Tieveny LM, McPhee SJ, Papadakis MA, editors. Current medical diagnosis and treatment. 46th ed. New York: McGraw Hill 2007.

2. Francischetti IM, Seydel KB Monteiro RQ. Blood coagulation, inflammation, and malaria. Microcirculation 2008; 15:81-107.

3. WHO Expert Committee on malaria; Twentieth report 1998. Geneva, Switzerland 2000.

4. National Anti-Malaria program. New Delhi: Directorate of NAMP. Ministry of health and family welfare 2002.

5. Ansari S, Khoharo HK, Abro A, Khund Al, Qureshi F. Thrombocytopenia in plasmodium falciparum malaria. J Ayub Med Coll Abbottabad 2009; 21:145147.

6. Callender DM, Hsue G. Artesunate: Investigational drug for the treatment of severe Falciparum malaria in Hawai'i. Hawaii Med J 2011; 70:77-79.

7. Shaikh $Q H$, Ahmad SM, Abbasi A, Malik SA, Sahito $A A$, Munir SM. Thrombocytopenia in malaria. $J$ Coll Physicians Surg Pak 2009; 19:708-710.

8. Lacerda MV, Mourao MP, Coelho HC Santos JB. Thrombocytopenia in malaria: Who cares? Mem Inst Oswaldo Cruz 2011; 106:5.

9. Taylor WR, Widjaja H, Basri H, Ohrt C, Taufik T, Tjitra E, Baso S. Changes in the total leukocyte and platelet counts in papuan and non-papuan adults from northeast papua infected with acute plasmodium vivax or uncomplicated plasmodium falciparum malaria. Malar J 2008; 7:259.

10. Adam MB, Adam GK, Rayis DA, Elbashir MI, Adam I. Thrombocytopenia in pregnant women with Plasmodium falciparum malaria in an area of unstable malaria transmission in eastern. Sudan BMC Clinical Pathology 2012; 12:10.

11. Memon AR, Afsar S. Thrombocytopenia in hospitalized malaria patients. Pak J Med Sci 2006; 22:141-143. 
12. Lee GR, Foerster J, Leukens J, Paraskeras F, Greer JP, Rodgers GM. Wintrobe's clinical hematology. 10th (Ed). Bethseda, Maryland: Lippincott Williams and Wilkins 1999.

13. Brittin GM. Automated optical counting of blood platelets. Blood 1971; 38:422.

14. Kokan A, Bhoi S, Praksh V, Kakar S. Profound thrombocytopenia in plasmodium vivax malaria. Diagnostic Microbial infectious Disease 1999; 35:243244.

15. Lathia TB, Joshi R. Can hematological parameters discriminate malaria from nonmalarious acute febrile illness in the tropics. Indian J Med Sci 2004; 58:239244.

16. Miana RN, Walsh D, Gaddy C, Hongo G, Waitumbi J, Otieno L. Impact of plasmodium falciparum infection on haematological parameters in children living in Western Kenya. Malar J 2010; 93:4.
17. Jadhav UM, Patkar VS, Kadam NN. Thrombocytopenia in malaria-correlation with type \& severity of malaria. JAPI 2004; 52:615-618.

18. Shaikh QH, Ahmad SM, Abbasi A, Malik SA, Sahito AA, Munir SM. Thrombocytopenia in malaria. J Coll Physicians Surg Pak 2009; 19:708-710.

19. Bhandary N, Vikram GS, Shetty H. Thrombocytopenia in Malaria: A clinical study. Biomedical Research $2011 ; 22: 489-491$.

20. Zaid HA, Ghadban WK. A study of thrombocytopenia in hospitalized vivax malaria patients. Journal of Emergency Medicine, Trauma and Acute Care 2012; 2012:22.

21. Rodriguez-Morales AJ, Sanchez E, Vargas M, Piccolo C, Colina R, Arria M. Occurrence of Thrombocytopenia in Plasmodium vivax Malaria Clin Infect Dis 2005; 41:130-131.

\begin{tabular}{|c|c|c|c|}
\hline Sr. \# & Author-s Full Name & Contribution to the paper & Author =s Signature \\
\hline 2 & $\begin{array}{l}\text { Yasmeen Batool } \\
\text { Sabeen Fatima } \\
\text { Gulzaib Pervaiz } \\
\text { Naseem Akhter } \\
\text { Maliha Asif }\end{array}$ & $\begin{array}{l}\text { Study design, data collection, writing } \\
\text { the manuscript, formulation of } \\
\text { tables,statistical analysis,interpretation } \\
\text { of results. } \\
\text { Data collection, formulation of tablers } \\
\text { reviewed and approved the manuscript, } \\
\text { Statistical analysis, interpretation of } \\
\text { results, Reviewed and approved the } \\
\text { manuscript. } \\
\text { Data collection, formulation of tablers } \\
\text { reviewed and approved the manuscript, } \\
\text { Statistical analysis, interpretation of } \\
\text { results, Reviewed and approved the } \\
\text { manuscript. } \\
\text { Study design, data collection, writing } \\
\text { the manuscript, formulation of tables } \\
\text { reviewed and approved. } \\
\text { Data collection, formulation of tablers } \\
\text { reviewed and approved the manuscript, } \\
\text { Statistical analysis, interpretation of } \\
\text { results, Reviewed and approved the } \\
\text { manuscript. } \\
\text { Study design, statistical analysis, } \\
\text { result interpretation, manuscript writing } \\
\text { and revising it critically for important } \\
\text { intellectual content. }\end{array}$ & Mo \\
\hline
\end{tabular}

